\title{
Prácticas alternativas al modelo de salud occidental utilizadas por cuidadores de pacientes con neoplasia hematológicas
}

\author{
Alternative practices to the conventional healthcare \\ model used by caregivers of patients hematologic \\ malignancies
}

\section{Alternativas práticas ao modelo de saúde ocidental, utilizadas pelos cuidadores de pacientes com neoplasia hematológicas}

\author{
Claudica Patricia Casas Patarroyo ${ }^{1}$, Ana Julia Carrillo², Carmelo R. \\ Fuentes Restrepo ${ }^{3}$, Lorena M. Melgarejo ${ }^{4}$, Ruth A. Castiblanco ${ }^{5}$, \\ María H. Solano ${ }^{6}$
}

\begin{abstract}
${ }^{1}$ Médica especialista en Medicina Interna-Hematología. Profesora asociada programa de hematología. Correo electrónico: claudiapatriciacasas@gmail.com

${ }^{2}$ Enfermera Magister administración en salud. Profesora titular Facultad de enfermería. Investigadora grupo perspectivas del cuidado. Correo electrónico: ajcarrillo@fucsalud.edu.co

${ }^{3}$ Médico especialista en Medicina Interna-Residente de segundo año de Hematología. Correo electrónico: casiusco28@gmail.com

${ }^{4}$ Enfermera. $\mathrm{PhD}(\mathrm{c})$ en salud pública. Investigadora grupo perspectivas del cuidado. Correo electrónico: lpmesa1@fucsalud.edu.co

${ }^{5}$ Enfermera. MSc(c) en salud pública. Instructora asistente Facultad de Enfermería. Investigadora grupo perspectivas del cuidado. Correo electrónico: racastiblanco@fucsalud.edu.co

${ }^{6}$ Médica especialista en Medicina Interna-Hematología. Profesora titular programa de hematología. Correo electrónico: mhsolano@fucsalud.edu.co
\end{abstract}

Cómo citar este artículo en edición digital: Casas Patarroyo, C., Carrillo, A.J., Fuentes Restrepo, C.R., Melgarejo, L.M., Castiblanco, R.A. \& Solano, M.H. (2019). Practicas alternativas al modelo de salud occidental utilizadas por cuidadores de pacientes con neoplasia hematológicas. Cultura de los Cuidados

(Edición digital), 23(53). Recuperado de 2http://dx.doi.org/10.14198/cuid.2019.53.16

Correspondencia: Claudia Patricia Casas Patarroyo. Dirección: Calle 10, No. 18-75, Hospital San José. Servicio Hematología. Bogotá-Colombia. Fundación Universitaria de Ciencias de la Salud

Correo electrónico: claudiapatriciacasas@gmail.com

Recibido: 20/11/2018; Aceptado: 13/02/2019

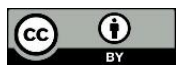

\section{ABSTRACT}

Introduction: Hematological neoplasms have the cultural burden of the word cancer which leads caregivers to look for different alternatives.

Objective: To analyse the anthropological link between care and tenderness to potentiate care as an essential "way to be" of the human being, who ontological way was born of care and needs care.

Methodology: Descriptive qualitative study. The participants were 17 caregivers, who were selected by convenience. Two discussion groups' sessions were 
performed. The sessions were recorded and transcribed in full. The analysis followed the Janice M. Morse framework.

Results: The findings that emerged permitted an approach toward understanding the practices used to care for patients affected by malignant hematological disease. The practices were organized in three types: 1. Spiritual practices: God's Will as determinant in the recovery process; 2 . Emotional Practices: the familiar ties make more bearable the illness and the treatment, and, 3. Corporal practices: origin products, vegetal, animal, semi-synthetic and no conventional pharmacological products.

Conclusion: The utilization of alternative practices is very common by caregivers. Healthcare practitioners cannot ignore this reality and need tools to adequately guide and inform patients and caregivers.

Keywords: Complementary therapies, culture, hematology, neoplasms, qualitative research, caregivers.

\section{RESUMO}

Introdução: As neoplasias hematológicas têm carga cultural da palavra câncer, levando aos cuidadores das pessoas que o sofrem a procurar diversas alternativas.

Objetivo: Descrever as práticas consideradas alternativas ao modelo de saúde Ocidental usado para cuidados de saúde, a partir da experiência de cuidadores de pacientes adultos com doenças hematológicas malignas no servicio de Hematologia Hospital San José de Bogotá.

\section{Metodologia: Estudo qualitativo} descritiva. Participaram 17 cuidadores de pacientes adultos com neoplasias hematológicas, seleccionados por conveniência dos registos do servicio Hematologia. Como uma técnica de coleta de informações dois grupos de discussão foram realizadas. As sessões foram gravadas e transcritas em sua totalidade. A análise foi feita de acordo com a proposta Janice $M$ Morse e se efectou uma triangulação foi realizado por pesquisadores.

Resultados: Surgiram três tipos de práticas utilizadas para cuidar de pessoas afectadas pela doença hematológica maligna; 1 . As práticas espirituais: a vontade de Deus tão influente na recuperação;. 2. práticas Affective: laços familiares tornar mais suportável a doença e o tratamento, e 3. práticas corporais: produtos de origem vegetal, animal, semi-sintético e farmacológico não convencional.

Conclusão: Os cuidadores de pessoas com câncer muitas vezes usam alternativa às práticas convencionais de tratamento, assim, a equipe de saúde não pode ignorar esta realidade e precisa de reforçar a orientação aos pacientes e seus cuidadores.

Palavras chave: Cuidadores, terapias complementares, neoplasias, hematologia, práticas de cuidado.

\section{RESUMEN}

Introducción: Las neoplasias hematológicas tienen la carga cultural de la palabra cáncer lo cual lleva a los cuidadores a buscar diversas alternativas.

Objetivo: Describir las prácticas consideradas alternativas al modelo de salud occidental utilizadas para el cuidado de la salud, desde la experiencia de los cuidadores de pacientes adultos con neoplasias hematológicas en un Servicio de Hematología.

Metodología: Estudio cualitativo descriptivo. Participaron 17 cuidadores de pacientes adultos con neoplasias hematológicas, seleccionados por conveniencia del registro del servicio de 
Hematología. Como técnica de recolección se realizaron dos grupos de discusión. Las sesiones fueron grabadas y transcritas en su totalidad. El análisis se hizo de acuerdo con la propuesta de Janice M. Morse y se efectuó triangulación por parte de investigadores.

Resultados: Emergieron tres tipos de prácticas. 1. Prácticas espirituales: la voluntad de Dios como influyente en la recuperación.; 2. Las prácticas afectivas: los lazos familiares hacen más llevadera la enfermedad y el tratamiento, y 3. Prácticas Corporales: productos de origen, vegetal, animal, semisintético y farmacológicos no convencionales.

Conclusión: Los cuidadores de la población con Cáncer utilizan prácticas alternativas al tratamiento convencional, por tanto, el equipo de salud no puede ignorarlas y necesita fortalecer la orientación al paciente y sus cuidadores.

Palabras clave: Cuidadores, Terapias complementarias, Neoplasias, Hematología, Prácticas de cuidado.

\section{INTRODUCCIÓN}

El cáncer representa un padecimiento físico y un estado de ansiedad ante los diferentes cambios que se generan a partir del diagnóstico, el tratamiento prolongado y los efectos asociados; para Barros y Nunes implica una "interrupción biográfica" y una incesante búsqueda de "respuestas" que lleva a la persona y su familia a aferrarse a diversas estrategias, prácticas y tratamientos que en algunas ocasiones pueden diferir de lo que ofrece la medicina convencional (de Barros y Nunes, 2006). Esta actitud de conservación del bienestar es un fenómeno que hace parte de la naturaleza humana; al respecto, Arévalo y cols. explican que cuando las personas experimentan una alteración en su bienestar, acuden a diversas “terapias, acciones o prácticas de carácter más científico (medicina convencional) o no, con el único objetivo de prevenir, mejorar o remediar las enfermedades" (Arévalo, Weng \& Jiménez, 2011, p. 138), a su vez, están influenciadas por la cultura y las particularidades de cada contexto. La antropología de la salud, se ha encargado de estudiar estos fenómenos para explicar y comprender las concepciones, imágenes y representaciones de las culturas y grupos humanos sobre lo que ellos consideran males, así como los distintos sistemas terapéuticos emergentes a lo largo y ancho del mundo y de la historia (Aparicio, 2007).

La frecuencia de prácticas para el cuidado de la salud consideradas no convencionales, ha sido reportada en diferentes estudios (Richardson et al., 2000; Idoyaga y Luxardo, 2005; Sánchez y Venegas, 2010), razón por la cual la Organización Mundial de la Salud, intentó reconocerlas bajo el concepto de Medicina Tradicional y Complementaria (MTC) (WHO, 2013); sin embargo, para instituciones especializadas en el tema como el National Center for Complementary and Alternative Medicine (NCCAM), este concepto era reducido y no expresaba la diversidad ni el sentido real de este tipo de prácticas en pacientes con cáncer, por tanto propuso reconocerlas como "un conjunto diverso de sistemas, prácticas y productos médicos y de atención de la salud que no se consideran actualmente parte de la Medicina convencional" (NCCAM, 2010), al respecto desde la perspectiva del cuidado de enfermería, llama la atención que ésta definición no hace referencia al aspecto vivencial $y$ a la experiencia de la enfermedad, categorías centrales para la medicina tradicional, como lo explica Laplantine, citado por Aparicio: 
"Mientras la intervención médica oficial pretende únicamente proporcionar una explicación experimental de los mecanismos químico-biológicos de la enfermedad y de los medios eficaces para dominarlos, las medicinas populares aportan una respuesta integral a una serie de insatisfacciones -no solamente somáticas sino también psicológicas, sociales, espirituales para algunos, y existenciales para todos- que el racionalismo social no está dispuesto a incorporar" (Laplantine 1992: 352)" (Aparicio, 2007).

Así, las formas de vivir y entender las enfermedades determinan mucho de las prácticas que las personas usan, por tanto, una práctica no solo es una acción que se ejecuta de manera automática, sino la expresión de una relación social entre los procesos de socialización y los modos de incorporarlo o racionalizarlo por parte de las personas. De acuerdo con Chaumont (2015), “el sentido de las prácticas permite comprender la lógica que pone en marcha a los agentes sociales que las producen y que actúan en un tiempo y en un contexto determinado" (p. 29). De acuerdo con lo anterior, la exploración de prácticas de cuidado frente al cáncer y en éste caso específico de las enfermedades hematoncológicas implica una exploración subjetiva, y un reconocimiento de las mediaciones que la cultura, el proceso de socialización y el contexto tienen en el desarrollo y variedad de las mismas, como parte de comprensiones más globales sobre la salud. Así, el presente estudio buscó describir las prácticas consideradas alternativas al modelo de salud occidental utilizadas para el cuidado de la salud, desde la experiencia y voces de los cuidadores de pacientes adultos con neoplasias hematológicas en el Servicio de
Hematología del Hospital de San José de Bogotá.

\section{METODOLOGÍA}

Estudio cualitativo descriptivo. Los participantes fueron hombres y mujeres mayores de edad cuidadores de pacientes adultos con neoplasias hematológicas. El paso inicial fue contactar a los pacientes que llevaran más de tres meses con quimioterapia, quienes facilitaron los datos de sus cuidadores para ser invitados directamente. Según la experiencia de los Hematólogos después de los tres meses las personas ya han tenido una trayectoria que les posibilita un mejor conocimiento del diagnóstico y mayor exploración de prácticas alternativas o complementarias al tratamiento convencional. Además, en este tiempo se ha generado una socialización e interacción entre el grupo de pacientes y familiares en espacios como la sala de quimioterapia y en consulta externa que permite el intercambio espontáneo de experiencias.

Como técnica de recolección de información, se realizaron dos grupos de discusión, entendidos como una reunión intencionada que busca construir los discursos de los grupos en su cotidianidad (Callejo, 1998). Las sesiones se realizaron el año 2015, en un salón que garantizó la privacidad y tranquilidad de los participantes, con una duración promedio de dos horas, estuvieron constituidos por diez participantes en el primer encuentro y siete diferentes en el segundo. Para la conformación de los grupos se tuvo en cuenta la disponibilidad de tiempo de los participantes y la experiencia compartida como cuidadores; se procuró que fuesen heterogéneos y mixtos, de modo que se enriqueciera el intercambio de experiencias 
y se pudiesen explorar múltiples prácticas y representaciones. Los dos grupos contaron con un moderador y un relator. El moderador orientó la discusión con base en el guion elaborado a partir de la experiencia de los investigadores y literatura existente, que contenía 3 temáticas orientadoras, la primera relacionada con la experiencia de cuidado, la segunda exploró las prácticas específicas y la tercera los modos de realización y preparación de las mismas.

El análisis de la información se realizó de acuerdo con lo propuesto por Morse (2003). Cada uno de los investigadores hizo la lectura línea a línea para extraer los códigos y organizarlos en una matriz junto con su interpretación; posteriormente los investigadores se reunieron para realizar el análisis conjunto, como mecanismo de triangulación. Cuando no existía consenso se contó con la participación de un experto en investigación cualitativa quien medió la discusión y facilitó despejar las dudas. Una vez establecidos los acuerdos en términos del análisis de los datos, estos fueron organizados en tres tipos de prácticas. Con respecto al rigor metodológico se realizó un proceso de triangulación con los participantes en los grupos de discusión, como forma de "devolución" de los resultados y como estrategia para asegurar la credibilidad de las interpretaciones realizadas (Castillo y Vásquez, 2003) por ello una vez conformadas las categorías se envió la información a cada uno de los participantes en forma personal o vía correo electrónico. Otra estrategia para mantener el rigor metodológico fue la grabación de las sesiones, previa firma del consentimiento informado de cada uno de los participantes, así como la transcripción literal de cada uno de los grupos focales por una persona experta, quien firmó un acuerdo de confidencialidad. Con el fin de mantener el anonimato se omitieron los nombres de los participantes. El análisis de la información se realizó de manera conjunta con la recolección con el fin de complementar o aclarar información sobre las prácticas. En el segundo grupo se alcanzó la saturación de los datos.

Las consideraciones éticas respetaron la Resolución 8430 de 1993 para investigación con seres humanos del Ministerio de Salud de Colombia, que clasifica esta investigación como de riesgo mínimo. El formato de consentimiento informado fue firmado por todos los participantes, previa explicación y comprensión del objetivo y los aportes del estudio a las personas afectadas por neoplasias oncológicas. El proyecto fue aprobado por el Comité de Investigación de la Facultad de Medicina y el de Ética e Investigaciones en Seres Humanos del HSJ y de la Fundación Universitaria de Ciencias de la Salud. Los archivos quedaron bajo custodia del coordinador del proyecto y la información obtenida solo fue utilizada para los fines de esta investigación. Se tuvo en cuenta, como consideración especial, que la participación de los cuidadores informales contara con aprobación de los pacientes ya que el tema de los grupos de discusión fueron las prácticas utilizadas para cuidarlos.

\section{RESULTADOS}

La edad de los participantes osciló entre 26 y 72 años (mediana: 48 años); 9 mujeres y 8 hombres. Respecto al nivel de estudios, 9 personas reportaron como máximo nivel educativo el bachillerato, el parentesco más frecuente fue ser esposo(a) (59.2\%). La mayoría de los cuidadores procedían de Bogotá D. C. (64.7\%). Del análisis de la información emergieron diferentes temas que luego de relacionarlos dieron origen a 
tres categorías que en conjunto permiten acercarse a la comprensión de las prácticas que este grupo de cuidadores informales utiliza.

Los discursos de los participantes permitieron identificar que las diversas prácticas utilizadas para el cuidado de personas con neoplasias hematológicas están ligadas a la fe que se les imprime. La fundamentación religiosa o espiritual fue concebida como factor determinante en los resultados de la acción independiente de las prácticas que se realicen. Para facilitar la descripción y comprensión de las mismas se integraron de acuerdo con sus características, orígenes y la dimensión humana que involucran, con base en lo cual se construyen tres tipos de prácticas: espirituales, afectivas y corporales. Es importante mencionar que la quimioterapia prevalece como principal tratamiento y en ningún momento se abandona, aun considerándola difícil para el paciente, contrario a lo que se presentó con algunas prácticas no convencionales, cuya permanencia y valoración como positivas o negativas dependen de los resultados inmediatos o de los efectos secundarios. En general, su ejecución implica un proceso de negociación entre el cuidador y el sujeto de cuidado, pues existen momentos en los que no hay convicción $\mathrm{y}$ se acude incluso al chantaje emocional, por parte del cuidador como situación límite en pro de lograr la mejoría de su familiar:

"¿mi amor tú no me quieres?, le decía, ¿no quieres a tu hija, a tu esposa?, me tocaba intimidarlo [para que se aplicara un medicamento veterinario]... mira tu tía hace 10 años se puso esto, no se ha hecho quimio, y sigue viviendo" G1C10-9.

\section{Prácticas espirituales: la voluntad de Dios como factor influyente en la recuperación.}

Esta categoría da cuenta de las diversas acciones relacionadas con la dimensión espiritual a las que acuden todos los cuidadores. Así el acercamiento y la comunicación con un ser supremo a través de la oración fue la práctica más común:

'me pegué a Dios de manera muy especial, le dije a ella [sujeto de cuidado] vamos a salir adelante" G1C6-52.

Asimismo, la idea de la voluntad de Dios es constante y prima sobre cualquier otra práctica, porque a ella se le atribuye el resultado de los tratamientos y el poder de curación como resultado de la misma fe:

"tengo que dejar todo en manos de Dios, en manos del médico, que se haga el proceso como ellos digan, creo que Dios les dio sabiduría para que eligieran bien" G1C5-51.

Por último, esta práctica espiritual resultó ser un mecanismo de apoyo que fortalece el vínculo familia-paciente $\mathrm{y}$ facilita el afrontamiento de la enfermedad:

"Nos hemos unido muchísimo alrededor de la oración" G2C3-73.

En los grupos de discusión se evidenció que las creencias religiosas y espirituales siempre estaban ligadas al paradigma Judeo-cristiano.

\section{Las prácticas afectivas: los lazos familiares hacen más llevadera la enfermedad y el tratamiento}

Estas prácticas incluyen las interacciones y relaciones intrafamiliares y con el equipo de salud. Se establecen y movilizan lazos afectivos que hacen llevadero el transcurso de la enfermedad y el tratamiento, involucran emociones que con el trascurrir 
del tiempo se convierten en sentimientos partiendo de que la experiencia de los cuidadores como lo expresó uno de ellos "puede ser tan dura que enferma" por ello recurren a estrategias como: la búsqueda de apoyo familiar, el amor, el aprender de otras experiencias y solidarizarse con los efectos secundarios del tratamiento. Estas prácticas posibilitan una mayor concienciación de la enfermedad y aceptación del tratamiento:

"tenemos un tío que está en el mismo proceso, entonces mi prima se ha vuelto experta y ha sido un gran apoyo, porque cuando uno no sabe, está perdido" G1C1-30.

Enlazadas con las prácticas anteriores están las actitudes frente al diagnóstico y al transcurso de la enfermedad, que surgen de manera espontánea y son similares entre los participantes, en la mayor parte de ellos su actitud estuvo ligada al ocultamiento del diagnóstico, su gravedad y a demostrar fortaleza frente al paciente para protegerlo emocionalmente; se asume una doble actitud que parece contradictoria, sin embargo, desde la experiencia de cuidar al ser querido, tiene una lógica fundamentada en el bienestar del otro, es decir, por un lado los cuidadores expresan sufrimiento, aflicción, angustia, incertidumbre, negación, ocultamiento, manipulación y temor a la muerte, y por otro se presentan ante su familiar enfermo como una columna de sustentación:

"cuando mi papá me pidió que le leyera el resultado del examen, yo iba leyendo, pero me volaba [ocultaba] la palabra cáncer" G1C5-2.

"yo le dije vamos a salir de esto, y lloraba donde ella no me viera" G1C6-4.

"tan pronto le aplicaron la quimioterapia, traje la rasuradora, nos sentamos en la habitación y nos peluqueamos juntos, porque esto lo vamos a llevar los dos" G2C2-44.
El miedo y el acercamiento a la muerte, relacionado con la visión que culturalmente se ha construido sobre el cáncer, juegan un rol importante en las prácticas de los cuidadores; así, emerge en los discursos un sentido de aislamiento social para limitar los prejuicios negativos que personas ajenas a la familia pueden dar al proceso. Además se suman múltiples interrogantes generados durante la trayectoria de la enfermedad, tanto en cuidadores como en pacientes; así, la búsqueda de respuestas es una constante y las fuentes se diversifican, pues no es suficiente la explicación convencional y acuden a diferentes medios para obtener información que alivie la incertidumbre, es a partir de éste proceso que la diada cuidadorpaciente considera importante combinar y probar varias alternativas de tratamiento y cuidado, que consideran favorables:

"La palabra leucemia alborota a los demás, empiezan a decir: ¡uy! tal persona se murió, entonces es mejor alejarse de todo lo que dice la gente" G2C9-53.

"Lo llevé donde un médico de la corriente bioenergética, él no le dio medicamentos, pero la manera en que le habló, le generó una actitud positiva" G2C1-58.

De este modo, a pesar de la interpretación que hacen del cáncer como un sinónimo de muerte, las perspectivas y la actitud de los cuidadores se van modificando lo largo de la quimioterapia, pues, aunque inicialmente para la mayoría los efectos secundarios representaron un deterioro evidente de las condiciones físicas y emocionales del paciente, después al observar resultados favorables, la perspectiva cambió, lo cual facilitó la adherencia al tratamiento e incluso generó vínculos afectivos con el personal de salud:

"A mi esposa la veo bastante bien, inicialmente fue demasiado duro, la 
movilizaban en silla de ruedas, ella no tenía fuerza ni para levantar su cabeza" G2C8-50.

En este estudio el amor fue el sentimiento que predominó como práctica reconfortante y de soporte para los pacientes y de compromiso para los cuidadores. Así lo expresaron:

"le dije lo que pasaba y juntos al piso totalmente; pero nos levantamos...y nos levantamos por el amor que nos tenemos" G1C2-14.

\section{Prácticas corporales: utilizan productos de origen, vegetal, animal, semi-sintético y farmacológicos no convencionales}

Esta categoría integra las diversas prácticas corporales utilizadas por los participantes las cuales tienen materialidad en el mundo físico y cuyos resultados se reflejaron en la mejoría biológica, aunque no se desliga del componente emocional y de fe al ejecutar la acción. Los participantes reportan el uso individual o combinado de productos de origen vegetal, animal, semisintéticos y farmacológicos no convencionales, para fortalecer el sistema inmunológico o "las defensas", y para potenciar la quimioterapia, los cuidadores comentan que en ningún momento los pacientes han manifestado el deseo de abandonarla y es considerada como el tratamiento convencional, ellos expresan que los productos "alternativos" actúan como coadyuvantes del tratamiento. Todos los cuidadores manifestaron administrar a quienes cuidan Agraz, Aloe Vera y algunos utilizan Arándanos, guanábana, Uva Isabela, Noni, Mangostino, Equinacea $\mathrm{y}$ Moringa:

'usamos aloe vera, (sábila) también hemos usado mangostino en fruta y preparo jarabe de noni. Lo conozco hace 10 años por una amiga, me dijo que era muy bueno para las defensas" G2C1-66.

"lo único es agraz y arándanos... el arándano es caro [costoso,] no sé si es mito que sube las defensas...además come durazno, pera, mandarina y guanábana" G2C8-98.

"de internet aprendí la terapia de Gerson, es zanahoria con manzana en extracto, no se debe licuar, preparo 2 zanahorias y 2 manzanas...pienso que ha funcionado" G2C3-74.

En la descripción de las experiencias se evidencia que la mayoría de prácticas se trasmiten a modo de tradición oral, y otras son conocidas a partir de medios de comunicación como internet; en algunos casos a pesar de no tener certeza de su efectividad la práctica se ejecuta, lo cual está relacionado con la necesidad de contribuir a que el sujeto de cuidado mejore su salud o tenga mejores condiciones de bienestar. Así lo expresó una cuidadora:

"Es una receta que comparten por todo lado, como curadora del cáncer, promovida por un sacerdote; podríamos decir que es milenaria, habla del poder del aloe vera, y su preparación es con un licor, puede ser whisky..." G2C1-69.

El anterior código permite ver las diferentes dimensiones que involucra una práctica corporal: el componente de la Fe, la finalidad, los materiales que se deben usar, la preparación y el argumento que subyace en la acción de cuidado. Frente a la preparación, conservación e indicación específicas sobre las prácticas corporales hubo amplia participación, fue posible identificar que habían apropiado esos conocimientos y hacen énfasis en las medidas higiénicas:

'Tiene que ser un frasco oscuro café o verde, que no le pase la luz, y mantenerlo en la nevera" G1C11-75. 
Los productos de origen animal que refirieron usar fueron: el chulo o gallinazo negro, el zuro o paloma y el pescado. Las formas de preparación mencionadas fueron diversas e incluyeron, caldos, guisos y otros métodos como la deshidratación y la pulverización. Este tipo de productos son los de mayor controversia a la hora de utilizarlos por parte del cuidador y del sujeto de cuidado. Algunos textos lo narran así:

"El chulo, creo que se lo tomó dos veces, la verdad fue una experiencia [cara de desagrado]... horrible, porque sabe muy feo y por las náuseas no... No lo volvió a probar. Los huesos se secaban al sol, se molían y en el caldito le echaban ese polvito" G2C6-134.

Diferentes productos de origen animal utilizados para el mejoramiento del estado de salud de las personas con cáncer se encuentran en el mercado en forma semisintética y los cuidadores al enterarse de su existencia los prefieren, dentro de los más usados mencionaron: el EMBRIOVIT® el guala-chulo y el calostro, en presentaciones de pastillas o cápsulas:

'El EMBRIOVIT®... es embrión de pato es de un laboratorio reconocido, se tomaba antes o después de las quimioterapias" G2C6-91.

"unas pastillas que las hace un científico a partir de un pájaro que se llama guala, yo creo que es el mismo chulo; le di como 3 frascos de 100 cápsulas" G1C7-88.

En uno de los grupos se mencionó el uso de suplementos alimentarios que habían sido formulados en otro momento de la vida del paciente o a un familiar para mejorar las condiciones nutricionales, y se considera tratamiento convencional:

"nosotros no le damos nada alternativo le suministramos un suplemento alimenticio, el ENTEREX® son 2 cucharadas y le ha servido" G2C8-94.
En este grupo de prácticas se comentó el uso de fármacos no autorizadas para el consumo humano, justificada a partir de su efectividad en personas con la misma enfermedad. Así lo describe un participante:

"Se llama VetalOG®...es una inyección muy buena, tiene casi las mismas indicaciones de la quimio, hay que mantenerlos bien alimentados, porque contiene mucho potasio $y$ la persona se debilitar mucho. A mi hijo le pusimos 3 frascos de $10 \mathrm{~cm}$, cada tercer día, centímetro y medio, Intramuscular" G1C10-64.

La cuidadora comentó que fue un campesino quien recomendó el uso a un médico que tenía cáncer, luego él se lo recomendó a la hermana de la cuidadora, debido a la percepción de la efectividad que tuvo en él; llama la atención como las prácticas de cuidado no derivadas del tratamiento convencional rápidamente se difunden, sin tener en cuenta posibles efectos secundarios, sobre los cuales a pesar de que no se indagó, emergieron espontáneamente medidas para controlarlos:

"Coge 2 pencas de sábila, la lava bien que no vaya a destilar salvia amarilla porque ese es el tóxico de la sábila, después lo corta y lo licua con 3 copitas de whisky" G1C11-74.

"Se le hacía anamú en infusión y se la tomaba, pero la tía que está en el pueblo le decía: el anamú no lo puedes tomar en exceso porque te hace bajar de peso" G1C5-84.

\section{DISCUSIÓN}

El estudio permitió identificar prácticas alternativas al tratamiento convencional de las enfermedades hematooncológicas las cuales son frecuentes, y están presentes en el imaginario social y en la realidad, es de resaltar que se combinan no solo entre ellas sino con el tratamiento convencional. 
Según lo expresado por los cuidadores suele no comentarse su uso con el equipo de salud; no es claro si esto se da, porque no se indaga al respecto o se prefiere ocultar. La complementariedad entre los diferentes tipos de prácticas ha sido reportada anteriormente en estudios como el de Chaumont, sobre la Salud Mental y las Prácticas No Convencionales, en el cual los participantes relataron como las combinaban con el fin de obtener mejores estados subjetivos de bienestar psíquico (Chaumont, 2015). Si bien es cierto el objetivo del estudio era explorar las prácticas, los resultados mostraron que las mismas se entretejen con las experiencias y creencias sobre la salud y la enfermedad, es de resaltar que siempre se fundamentan en la fe de que van a funcionar y aunque pueden existir dudas sobre su efectividad, bien sea en el paciente o en el cuidador el deseo de que el sujeto de cuidado se cure las supera. Las practicas descritas tienen como fin hacer algo que ayude al tratamiento convencional, pero se abandonan si no se ven resultados de mejoría de forma inmediata o si los "efectos secundarios" son superiores a la voluntad de las personas, como ocurre con el caso del chulo, dónde las "nauseas" y la sensación de desagrado son muy incomodas. El uso de las prácticas descritas en esta investigación, es similar a lo reportado en estudios desarrollados en estados Unidos, México y Argentina (Idoyaga y Luxardo, 2005; Gerson, Serrano y Villalobos; 2006). Así mismo las prácticas religiosas que emergieron han sido reportadas por autores como Aquino y cols. (2007), como una estrategia para sobrevivir al cáncer, pues la creencia en un ser supremo representa la posibilidad de curación en diversas culturas.

Dentro de las frutas más usadas para mejorar el sistema inmunológico se encontraron los arándanos y el agraz, que hacen parte de las denominadas Blueberries (Lohachoompol, Srzednicki y Craske, 2004), se asocian con un alto contenido de antocianinas y antioxidantes que actuarían, de acuerdo con el estudio de Maldonado y cols. (Maldonado, Arango y Rojano, 2014), como citotóxico y antiproliferativo. En esta misma línea, Chen y cols. (2014), demuestran como el aloe vera posee alto contenido en antraquinonas, a las cuales se les atribuye una acción antitumoral generada por la interrupción del ciclo celular e inducción de la apoptosis, adicionalmente se demostró efectos antimetastásico, antiangiogénico y de fortalecimiento del sistema inmune. Por su parte el mangostino (Garcinia mangostana) y la guanábana (Annona muricata) han sido analizadas en su potencial anticancerígeno entre otros por autores como Pieme, Chen, Akao, Fekam y col. (2014), quienes asocian este efecto al alto contenido de flavonoides, fenoles y la relación con niveles elevados de anomuricina $\mathrm{E}$ que actuarían sobre la caspasa3 de tumores no sólidos como la leucemia promielocítica y la leucemia mieloide crónica. Para el caso del Noni (Morinda Citrifolia), Liu y cols. (2001), describen el potencial efecto antiproliferativo por altas concentraciones de 2 glucósidos conocidos como la 6-O-(bD-glucopiranosil)-1-O-octanoyl-b-D glucopyranose y el ácido asperulosidico, que suprimen el 12-Otetradecanoilphorbol-13-acetato (promotor tumoral) y el factor de crecimiento epidérmico a nivel de las células tumorales. Respecto al EMBRIOVIT® $\mathrm{y}$ otros suplementos mencionados no se conocen estudios científicos sobre su efecto en las células cancerígenas. 
Referente al consumo de Chulo o gallinazo negro (Coragyps atratus), Sánchez y cols. (2012), describieron la ingesta de sangre fresca del animal como práctica popular para la patología hematopoyética maligna, y al igual que en este estudio, los cuidadores le atribuían la capacidad de potenciar el sistema inmunológico. Respecto al fármaco no convencional que emergió en la experiencia de los cuidadores cuya marca comercial es VETALOG® (acetato de triamcinolona), es un medicamento de uso veterinario, pertenece a los corticoides de acción intermedia, no se encontró evidencia en la literatura científica sobre su uso en humanos y valdría la pena alertar a pacientes y cuidadores sobre los riesgos que conlleva el uso simultáneo de varios corticoides como los que se utilizan de manera concomitante a la quimioterapia, además de las diferencias en la preparación química y en la concentración de los medicamentos para humanos y los productos para animales.

\section{RECOMENDACIONES}

La información proporcionada por los participantes hace evidente la necesidad de que el equipo de salud tratante sea consciente de las prácticas utilizadas por los cuidadores de los pacientes con el fin de incluir en los planes de atención intervenciones encaminadas a disminuir las posibles interacciones negativas con el tratamiento convencional e informar de las mismas a los pacientes y cuidadores. Más allá de la intención de validar el discurso de los participantes respecto al uso de productos farmacológicos no convencionales para el cuidado del cáncer desde la literatura biomédica, el grupo investigador considera relevante aproximarse a estudios sobre los posibles mecanismos de acción de los diferentes productos utilizados por los cuidadores; además los resultados hacen evidente la necesidad de profundizar desde la investigación básica en muchos de los productos utilizados, pues es una realidad su uso frecuente y cotidiano.

Con Respecto a algunos productos se encontró en la literatura revisada estudios que sugieren la posibilidad de un efecto anticancerígeno, pero no se conocen las posibles interacciones con la terapia convencional, a la cual nunca renuncian los pacientes; de modo que es recomendable desarrollar investigación que permita aproximarse a las mismas.

\section{AGRADECIMIENTOS}

El grupo investigador agradece de manera especial a los cuidadores que contribuyeron con sus relatos para la realización de este estudio y a los pacientes que facilitaron los datos para contactarlos, igualmente a Celmira Laza Vásquez por su participación en el diseño de la propuesta que origino los resultados que hoy se presentan.

\section{BIBLIOGRAFÍA}

- Aparicio Mena, A. (2007). La antropología aplicada, la medicina tradicional y los sistemas de cuidado natural de la salud. Una ayuda intercultural para los padecimientos crónicos. Gazeta de Antropología, 23, 14.

- Aquino, V.V. \& Zago, M.M. (2007). The meaning of religious beliefs for a group of cancer patients during rehabilitation. Rev Lat Am Enfermagem, 15(1), 42-7.

- Arévalo de Andrade, M., Weng, H., Nien, T. \& Jiménez Herrera, L. (2011). Terapias, prácticas y acciones en la búsqueda de un mejor estado de salud-enfermedad. Revista Costarricense de Salud Pública, 20(2), 137-144.

- Callejo Gallejo J. (1998). Articulación de perspectivas metodológicas: posibilidades del grupo de discusión para una sociedad reflexiva. 
Rev Sociología, 56, 31-5.

- Castillo, E. \& Vásquez, M. (2003). El rigor metodológico en la investigación cualitativa. Colombia Médica, 34(3), 164-167.

- Chaumont, D. (2015). Salud Mental y Prácticas No Convencionales: Aproximaciones acerca del uso de prácticas consideradas alternativas o complementarias al modelo sanitario occidental, desde la perspectiva de los sujetos que las utilizan. (Tesis para optar por el título de Maestría en salud mental). Bogotá: Universidad Nacional de Córdoba. Facultad de Ciencias Médicas Facultad de Psicología.

- Chen, R., Zhang, J., Hu, Y., Wang, S., Chen, M. \& Wang, Y. (2014). Potential antineoplastic effects of Aloe-emodin: a comprehensive review. Am J Chin Med, 42(2), 275-88.

- De Barros, NF. \& Nunes, ED. (2006) Complementary and alternative medicine in Brazil: one concept, different meanings. Cad Saude Pública, 22(10), 2023-8.

- Idoyaga Molina, A. \& Luxardo, N. (2005). Medicinas no convencionales en cáncer. Medicina, 65(5), 390-394.

- Lohachoompol, V., Srzednicki G. \& Craske J. (2004). The Change of Total Anthocyanins in Blueberries and Their Antioxidant Effect After Drying and Freezing. J Biomed Biotechnol, 5, 24852.

- Maldonado Celis, M.E., Arango Varela, SS. \& Rojano, BA. (2014). Free radical scavenging capacity and cytotoxic and antiproliferative effects of Vaccinium meridionale Sw. agains colon cancer cell lines. Rev Cubana Plant Med, 19, 2, 172-184.

- Morse, J.M. (Ed). (2003). Asuntos críticos en los métodos de investigación cualitativa. Medellín. Colombia: Universidad de Antioquía.

- National Center for Complementary and Alternative Medicine. (2010). Uso de la medicina complementaria y alternativa para el cáncer. Estados Unidos. NY: Departamento de Salud y Servicios Humanos de los Estados Unidos (HHS).

- Pieme, C.A., Kumar, S.G., Dongmo, M.S., Moukette, B.M., Boyoum, F.F., Ngogang, J.Y., et al. (2014) Antiproliferative activity and induction of apoptosis by Annona muricata (Annonaceae) extract on human cancer cells. BMC Complement Altern Med, 14, 516.

- Richardson, M.A., Sanders, T., Palmer, J.L., Greisinger, A. \& Singletary, SE. (2000). Complementary/alternative medicine use in a comprehensive cancer center and the implications for oncology. J Clin Oncol, 18 (13), 2505-14.

- Sánchez Pedraza, R., Gamba Rincón, M.R. \& González Rangel, A.L. (2012). Use of black vulture (Coragyps atratus) in complementary and alternative therapies for cancer in Colombia: a qualitative study. J Ethnobiol Ethnomed, 8(1), 20.

- Sánchez, R. \& Venegas, M. (2010). Aproximaciones complementarias y alternativas al cuidado de la salud en el Instituto Nacional de Cancerología: estudio de prevalencia. Rev.Colomb.Cancerol, 14(3), 135-43.

- World Health Organization. (2013) The WHO Tradicional medicine strategy 2014-2023. Pekin: World Health Organization, pp. 1-70. 